\title{
Quantum cloning in spin networks
}

\author{
Gabriele De Chiara, ${ }^{1}$ Rosario Fazio, ${ }^{1}$ Chiara Macchiavello, ${ }^{2}$ \\ Simone Montangero, ${ }^{1}$ and G. Massimo Palma ${ }^{3}$ \\ 1 NEST-INFM \& Scuola Normale Superiore, \\ piazza dei Cavalieri 7, I-56126 Pisa, Italy \\ ${ }^{2}$ INFM $\&$ Dipartimento di Fisica “A. Volta”, Via Bassi 6,I-27100 Pavia, Italy \\ 3 NEST-INFM \& Dipartimento di Tecnologie \\ dell'Informazione, Universita' degli studi di Milano \\ via Bramante 65, I-26013 Crema(CR), Italy
}

(Dated: November 6, 2018)

\begin{abstract}
We introduce a new approach to quantum cloning based on spin networks and we demonstrate that phase covariant cloning can be realized using no external control but only with a proper design of the Hamiltonian of the system. In the $1 \rightarrow 2$ cloning we find that the XY model saturates the value for the fidelity of the optimal cloner and gives values comparable to it in the general $N \rightarrow M$ case. We finally discuss the effect of external noise. Our protocol is much more robust to decoherene than a conventional procedure based on quantum gates.
\end{abstract}

PACS numbers: 03.67.Hk,42.50.-p,03.67.-a 
Quantum information processing protocols [1] are typically described and analysed in terms of qubits and quantum gates, i.e. of quantum networks. Very recently increasing attention has been devoted to the possibility of implementing the desired task by a tailored design of a spin network and an appropriate choice of the couplings and the evolution time. Most notably in these schemes the couplings between qubits are fixed and this may turn to be an important advantage in the implementation of quantum protocols with solid state devices. Quantum computation for a spin network based on Heisenberg couplings was discussed in Ref. [2]. It was also shown that unmodulated Heisenberg chains can be used to transfer unknown quantum states over appreciable distances $\left(\sim 10^{2}\right.$ lattice sites $)$ with reasonably high fidelity [3, 4]. Even perfect transfer could be achieved over arbitrary distances in spin chains by a proper choice of the modulation of the coupling strengths [5], if local measurements on the individual spins can be implemented [6] or when communicating parties have access to limited numbers of qubits in a spin ring [7]. Together with the understanding of the dynamics of entanglement in spin systems [3, 4, 8], this approach to quantum communication may lead to the implementation of more complicated protocols such as entanglement swapping, teleportation or cloning, just to mention a few of them.

The aim of this Letter is to show that the dynamics of spin networks with fixed couplings can be applied successfully to the problem of quantum cloning. The no-cloning theorem 9$]$ states the impossibility to make a perfect copy of an unknown quantum state. This no-go theorem has profound implications not only at a fundamental level but also for practical reasons, since it is the key ingredient to guarantee the security of quantum cryptographic protocols [10]. Although perfect cloning is forbidden by the laws of quantum mechanics, it is of great interest to optimize the performance of approximate cloning machines. In a pioneering work in this direction a transformation for copying an unknown qubit state with a state-independent fidelity, known as $1 \rightarrow 2$ universal cloning, was presented [11]. It was later proved to be optimal [12]. The more general problem of $N \rightarrow M$ universal cloning, where $N$ copies of an unknown input pure state are cloned to $M$ output approximate copies, has been also addressed [13]. Notice that the fidelity of the cloning transformation can be increased if some prior partial knowledge of the states to be cloned is available. The first state-dependent cloner was proposed in [12], where cloning of two non orthogonal states was analysed. Another interesting example of non universal cloning, which we will consider in this paper, is the case of qubits lying on the equator of the Bloch sphere. This class of 
cloners was proposed in Ref. 14] and is known as the Phase Covariant Cloning (PCC).

Several protocols for implementing cloning machines have been already achieved experimentally [15, 16, 17, 18]. In all these proposals to clone a quantum state, the required set of operations is realized by means of quantum gates, or otherwise a post-selection of the state needs to be performed. (For example, the quantum network corresponding to the $1 \rightarrow 2$ PCC consists of two C-NOT gates together with a controlled rotation [19].) In the following we concentrate on the phase covariant case and we will address the question whether it is possible to perform optimal cloning without almost any external control. We will show that this is indeed possible by choosing a proper Hamiltonian and the topology of the spin network. In Fig.1 we give some examples. It turns out that it is then possible to clone a quantum state with very high fidelity, in some cases with optimal fidelity, by letting the system evolve for a given time lapse $t_{c}$. The choice of $t_{c}$ is the only control which we assume (and need) to have on the system. The results of this work are summarized in Figs 2 and 3. We will show that the time $t_{c}$ required for the protocol does not seem to systematically increase, for the case considered, as a function of $N$ and $M$. This is a great advantage over the conventional schemes if the unavoidable effect of decoherence is taken into account (see Fig 3).

The model Hamiltonian necessary to accomplish our task is given by

$$
H_{\lambda}=\frac{1}{4} \sum_{i j} J_{i j}\left(\sigma_{x}^{i} \sigma_{x}^{j}+\sigma_{y}^{i} \sigma_{y}^{j}+\lambda \sigma_{z}^{i} \sigma_{z}^{j}\right)+\frac{B}{2} \sum_{i} \sigma_{z}^{i}
$$

where $\sigma_{x, y, z}^{i}$ are the Pauli matrices, $J_{i j}$ are the exchange couplings defined on the links joining the sites $i$ and $j$ and $B$ is an externally applied magnetic field. In all the cases we consider in this work the couplings $J_{i j}$ are different from zero only if $i, j$ are nearest neighbours. To specify which couplings are non-zero one has to define the topology of the spin network. The anisotropy parameter $\lambda$ ranges from 0 (XY Model) to 1 (Heisenberg Model) [20]. Once the Hamiltonian in Eq.(11) is specified the cloning protocol consists in the following steps: i) the initialization of the network, where the states to be cloned are stored in $N$ sites and the remaining sites are in a blank state; ii) the evolution of the system (without any further manipulation) up to the time $t_{c}$ at which the state has been copied in the (initially) blank states.

$1 \rightarrow M$ Cloning - A natural choice, although not unique, for the graph to be used in this case is a star network as illustrated in Fig 1a. The spin in the state to be cloned is placed 
in the center of the star and will be labeled by 0 . The outer spins in the star, labeled from 1 to $M$, are the blank qubits on which the state will be copied. Note that the central spin plays a double role of the state to be cloned and that of the ancilla. The initial state of the network is

$$
|\Psi\rangle=\cos \frac{\vartheta}{2}|00 \ldots 0\rangle+e^{i \varphi} \sin \frac{\vartheta}{2}|10 \ldots 0\rangle
$$

$\left(|0\rangle\right.$ and $|1\rangle$ are eigenstates of $\sigma_{z}$ with eigenvalues \pm 1$)$.

Let us first consider the Heisenberg model $(\lambda=1$ in Eq.(10). We present the results only for $B=0$, as the presence of $B$ in this case just changes the time at which the maximum fidelity is achieved. The system can be considered as a spin-1/2 (placed in the central site) interacting with with a spin-S particle $S=M / 2$ via an exchange interaction $H_{\lambda=1}=J \overrightarrow{S_{0}} \cdot \vec{S}$ where $\vec{S}_{0}=\vec{\sigma}_{0} / 2$ and $\vec{S}=1 / 2 \sum \vec{\sigma}^{i}$ is the total spin of the outer sites. The energy eigenstates coincide with those of the total angular momentum $\vec{I}=\vec{S}_{0}+\vec{S}$, labeled as $\left|I, I_{z}\right\rangle$, and can be written in the computational basis using Clebsh-Gordan coefficients [21]. The eigenvalues can be found noting that $\overrightarrow{S_{0}} \cdot \vec{S}=1 / 2\left(I^{2}-S^{2}-S_{0}^{2}\right)$. After calculating the total state of the star at time $t$ we are interested in the reduced density matrix $\rho_{\text {out }}$ of one of the outer spins. Notice that the system is invariant under any permutation of the outer spins so their reduced density matrices are equal. This property guarantees the symmetry requirement of cloning machines, i.e. all the clones are equal. We evaluate the quality of the cloning transformation in terms of the fidelity $F=\left\langle\psi\left|\rho_{\text {out }}\right| \psi\right\rangle$ of each outer spin with respect to the initial state of the qubit to be cloned $|\psi\rangle$. The fidelity is optimized at times $t_{c}^{(M)}=\frac{2 \pi}{J(M+1)}$ where it takes the maximum value $\mathcal{F}_{\lambda=1}=\max _{t}\{F\}$

$$
\mathcal{F}_{\lambda=1}=\frac{4+(3+M)[M+(M-1) \cos \vartheta]-(M-1) \cos 2 \vartheta}{2(1+M)^{2}}
$$

The other interesting case to be considered (which turns out to be the optimal as compared with the Heisenberg model) is the XY model $(\lambda=0$ in Eq.(1) $)$. We are interested only in the eigenspaces with total angular momentum $(M \pm 1) / 2$ since the total angular momentum is a good quantum number. We define the eigenstates of angular momentum of the outer spins as $\left|j, m_{z}\right\rangle$ where $j$ and $m_{z}$ are the modulus and $z$ component of $\vec{S}$. In the subspace $j=M / 2$ the eigenstates and eigenvalues are $\left|\psi_{j, m_{z}}^{ \pm}\right\rangle=\frac{1}{\sqrt{2}}\left(|1\rangle\left|j, m_{z}\right\rangle \pm|0\rangle\left|j, m_{z}-1\right\rangle\right)$ $E_{ \pm}= \pm \frac{J}{2} \sqrt{\left(j+m_{z}\right)\left(j-m_{z}+1\right)}+B\left(m_{z}-\frac{1}{2}\right)$

for $m_{z}=j, j-1, \ldots,-j+1$. The other eigenstates are $|0\rangle|j, j\rangle$ and $|1\rangle|j,-j\rangle$ with eigenvalues $\pm B(j+1 / 2)$ respectively [22]. One can show that in the XY model the fidelity 
is maximized for $t_{c}^{(M)}=\frac{\pi}{\sqrt{M} J}$ and $B=\frac{J}{2} \sqrt{M}$ and the maximal fidelity is

$$
\mathcal{F}_{\lambda=0}=\frac{1+\sqrt{M}+2 M+2(M-1) \cos \vartheta-(\sqrt{M}-1) \cos 2 \vartheta}{4 M}
$$

In both cases of Eqs. (34) the fidelity does not depend on $\varphi$.

An analysis of Eqs.(34) allows to draw several conclusions along the outlined motivations of this work. Let us begin with the comparison of the XY versus Heisenberg cloning machines. In Fig 2 we show the results for the maximum fidelity for the XY and Heisenberg models for the $1 \rightarrow 2$ cloning as a function of $\vartheta$.

In this case the fidelity for the XY model coincides with that of the optimal PCC [14, 23]. We have thus demonstrated that for $M=2$ phase covariant cloning can be realized without external gates. Note that for $M=2$ and equatorial qubits $\mathcal{F}_{\lambda=1}=5 / 6$, i.e. it is exactly the value for the universal quantum cloner [11, 12]. The previous observation, together with the fact that the Heisenberg cloner is less accurate, shows that the choice of the model is crucial in order to realize the required protocol. For generic $M$ and for $\vartheta=\pi / 2$ the three fidelities are compared in the inset of Fig 2. The optimal PCC fidelity for generic $M$ was derived in Ref. 24] and it is reported for comparison in the same plot. By increasing $M$ the optimal fidelity scales differently for the two models, namely as $1 / M$ for the Heisenberg Hamiltonian and as $1 / \sqrt{M}$ for the XY model. In both cases the maximum fidelity is lower than that of the PCC. However one should note that for both models the time at which the maximum fidelity is achieved decreases with increasing number of copies. As we will show later, our protocol will be much more efficient in the (unavoidable) presence of decoherence.

Until now we concentrated our attention to the $1 \rightarrow M$ cloning using the spin star configuration. Obviously this is not a unique choice and one can analyse how the results presented up to now depend on the topology of the spin network. For the $1 \rightarrow M$ cloner we studied a configuration of the spins in tree graphs like the one depicted in Fig 1b. The initial state is placed at the root of the tree while the qubits, onto which the original state will be copied, are on the last level. Each graph is characterized by the number $k$ of links departing from each site and the number $j$ of intermediate levels between the top and the blank qubits level. Our results show that the fidelity is almost independent of the graph. For example, by considering the XY model and $\vartheta=\pi / 2, \mathcal{F}_{\lambda=0}=0.676$ for $M=8(k=2$, $j=2)$ while for $M=27(k=3, j=2) \mathcal{F}_{\lambda=0}=0.596$.

We also analysed the role of imperfections in the spin star network and considered the 
case in which the coupling constants are fixed in time but random. For $J_{1 i}$ in the interval $[0.9 \mathrm{~J} ; 1.1 \mathrm{~J}]$ the average fidelity (obtained sampling 500 realizations of disorder) decreases only by less than $0.2 \%$ as compared to the ideal cases.

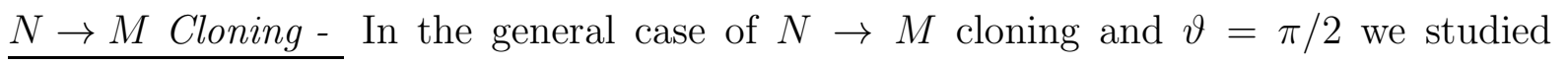
numerically the dynamics of the network shown in Fig 11. We considered systems up to 9 sites. We concentrated mostly on the case $\lambda=0$ which gave higher fidelities compared to the case $\lambda=1$. The results, for several values of $N$ and $M$ are shown in the Table प. The values of the fidelity and the times $t_{c}$ (at which the maximum fidelity is obtained) confirm the considerations expressed for the $1 \rightarrow M$ case. The simple cloning procedure we propose is able to attain fidelities which are comparable with the optimal fidelities for the $N \rightarrow M$ PCC. The time $t_{c}$ in this case is weakly dependent on $N$ and $M$. This means that the complexity of the protocol does not seem to increase with increasing number of qubits.

Cloning in the presence of Noise - The strength of the proposed protocol clearly emerges comparing the effect of decoherence on our protocol and on the known cloning quantum circuits [18, 25]. We model external noise as external fields $b_{i}(t)$ fluctuating independently on each spin of the network. The level of the noise is characterized by $\left\langle b_{i}(t) b_{j}\left(t^{\prime}\right)\right\rangle=\Gamma \delta_{i j} \delta\left(t-t^{\prime}\right)$ (we consider gaussian fluctuations and $\left\langle b_{i}(t)\right\rangle=0$ ). In Fig 3 we compare our results for the $1 \rightarrow 2$ and $1 \rightarrow 3$ cloning with the quantum circuits. In order to be consistent we implement the quantum gates of Refs. 18, 25] considering spins interacting through a time dependent $\mathrm{XY}$ couplings [26]. Even for a very low noise level $\Gamma / J \sim 10^{-3}$ our protocol is much more efficient than using quantum circuits. Moreover we believe that in a real implementation also the effective coupling to the environment can be different. Our system indeed may be decoupled from the environment during the evolution and coupled only at the measurement stage. The circuit model instead is always coupled to the environment because an active control on the Hamiltonian is always needed.

Implementation - The networks of Fig 1 and the XY Hamiltonian can be easily implemented by means of superconducting nanocircuits [27]. Qubits in the charge regime (represented by the sites in Fig (1) and coupled by Josephson junctions (represented by the links in Fig (1) realize the optimal PCC described in this work. The preparation of the initial state and the measurement procedure can be performed as described, for example, in Ref.[27]. Solid state cloning can be realized with present day technology.

This work was supported by the European Community under contracts IST-SQUIBIT, 
IST-SQUBIT2, IST-QUPRODIS, IST-ATESIT, and RTN-Nanoscale Dynamics.

[1] M. Nielsen and I. Chuang, Quantum Computation and Quantum Communication, (Cambridge University Press, Cambridge, 2000).

[2] S. C. Benjamin and S. Bose, Phys. Rev. Lett. 90, 247901 (2003); M.-H. Yung, D.W. Leung, and S. Bose, Quant. Inf. Comp. 4, 174 (2004).

[3] S. Bose, Phys. Rev. Lett. 91, 207901 (2003).

[4] V. Subrahmanyam, Phys. Rev. A 69, 034304 (2004).

[5] M. Christandl, N. Datta, A. Ekert, A. J. Landahl, Phys. Rev. Lett. 92, 187902 (2004).

[6] F. Verstraete, M.A. Martin-Delgado, J.I. Cirac, Phys. Rev. Lett. 92, 087201 (2004).

[7] T.J. Osborne, N. Linden, Phys. Rev. A 69, 052315 (2004).

[8] X. Wang, Phys. Rev. A 64, 012313 (2001); L. Amico, A. Osterloh, F. Plastina, R. Fazio, G.M. Palma, Phys. Rev. A 69, 022304 (2004) S. Montangero, G. Benenti, and R. Fazio, Phys. Rev. Lett. 91, 187901 (2003).

[9] W.K. Wootters and W.H. Zurek, Nature 299, 802 (1982)

[10] For a recent review on quantum cryptography see N. Gisin, G. Ribordy, W. Tittel, and H. Zbinden Rev. Mod. Phys. 74, 145 (2002).

[11] V. Bužek and M. Hillery, Phys. Rev. A 54, 1844 (1996).

[12] D. Bruß, D. P. DiVincenzo, A. Ekert, C. A. Fuchs, C. Macchiavello, J. A. Smolin, Phys. Rev. A 57, 2368 (1998).

[13] N. Gisin and S. Massar, Phys. Rev. Lett. 79, 2153-2156 (1997); D. Bruss, A. Ekert and C. Macchiavello, Phys. Rev. Lett. 81, 2598 (1998); R. Werner, Phys. Rev. A58, 1827 (1998).

[14] D. Bruß, M. Cinchetti, G. M. D’Ariano, C. Macchiavello, Phys. Rev. A 62, 012302 (2000).

[15] H. K. Cummins, C. Jones, A. Furze, N. F. Soffe, M. Mosca, J. M. Peach, J. A. Jones, Phys. Rev. Lett. 88, 187901 (2002).

[16] A. Lama-Linares, C. Simon, J.-C. Howell and D. Bouwmeester, Science 296, 712 (2002).

[17] D. Pelliccia, V. Schettini, F. Sciarrino, C. Sias and F. De Martini, Phys. Rev. A 68, 042306 (2003); F. De Martini, D. Pelliccia and F. Sciarrino, Phys. Rev. Lett. 92, 067901(2004).

[18] J. Du, T. Durt, P. Zou, L.C. Kwek, C.H. Lai, C.H. Oh, and A. Ekert, quant-ph/0311010.

[19] C.-S. Niu and R.B. Griffiths, Phys. Rev. A 60, 2764 (1999). 
[20] The model is invariant under rotations around the $z$ axis so it is a natural candidate for a PCC. We also analysed the case in which the XY symmetry is broken, the results for the fidelity were always much worse than for XY or Heisenberg models.

[21] To find the evolution for the initial state, Eq.(21), we need to express it in the eigenstate basis. After some simple algebra one finds that $|00 \ldots 0\rangle=\left|\frac{M+1}{2}, \frac{M+1}{2}\right\rangle$ and $|10 \ldots 0\rangle=$ $\frac{1}{\sqrt{1+M}}\left|\frac{M+1}{2}, \frac{M-1}{2}\right\rangle-\frac{\sqrt{M}}{\sqrt{1+M}}\left|\frac{M-1}{2}, \frac{M-1}{2}\right\rangle$.

[22] The initial state of the star is the same as for the Heisenberg model (2) and it can be written in terms of eigenstates of the Hamiltonian as $|\Psi(0)\rangle=\cos \frac{\vartheta}{2}|0\rangle|j, j\rangle+2^{-1 / 2} e^{i \varphi} \sin \frac{\vartheta}{2}\left[\left|\psi_{M / 2, M / 2}^{+}\right\rangle+\right.$ $\left.\left|\psi_{M / 2, M / 2}^{-}\right\rangle\right]$

[23] J. Fiurášek, Phys. Rev. A 67, 052314 (2003).

[24] G. M. D’Ariano and C. Macchiavello, Phys. Rev. A 67, 042306 (2003).

[25] V. Bužek, S.L. Braunstein, M. Hillery and D. Bruß, Phys. Rev. A 56, 3446 (1997).

[26] We refer to N. Schuch and J. Siewert, Phys. Rev. A 67, 032301 (2003)for the implementations of these circuits with time dependent XY model.

[27] Yu. Makhlin, G. Schön, and A. Shnirman, Rev. Mod. Phys. 73, 357 (2001). 


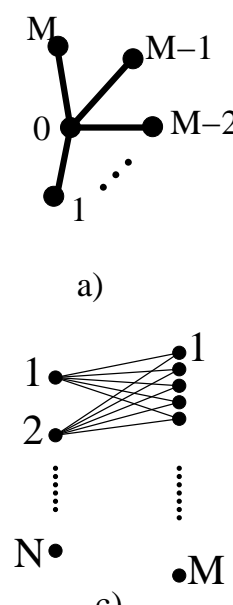

c)

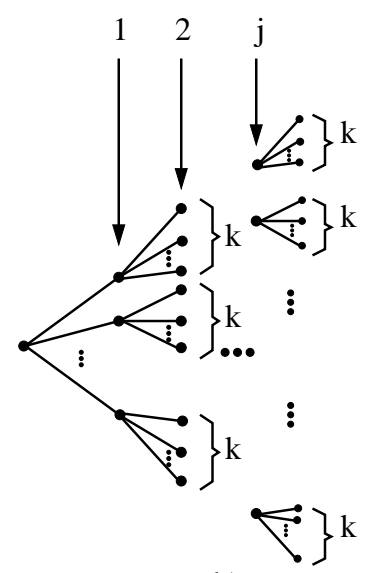

b)

FIG. 1: Different topologies for $N \rightarrow M$ cloner: a)Spin star network for $1 \rightarrow M$ cloner. b)Generic graph for the $1 \rightarrow M$ cloner with $j$ intermediate steps and $k$ links departing from each vertex. c) Spin network for the $N \rightarrow M$ cloning.

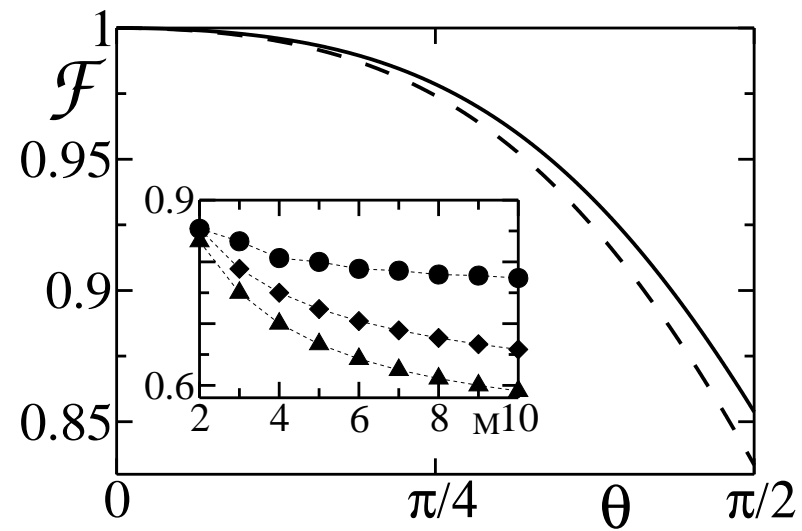

FIG. 2: The fidelities $\mathcal{F}_{\lambda}$ for $M=2$ for generic $\vartheta$ for the XY (solid) and Heisenberg (dashed) model are shown. Notice that the optimal fidelity for the PCC is exactly that of the XY model. Inset: the fidelity $\mathcal{F}$ for the three cases PCC (circle), XY (diamond) and Heisenberg (triangle) as functions of $M$ for $\vartheta=\pi / 2$. 


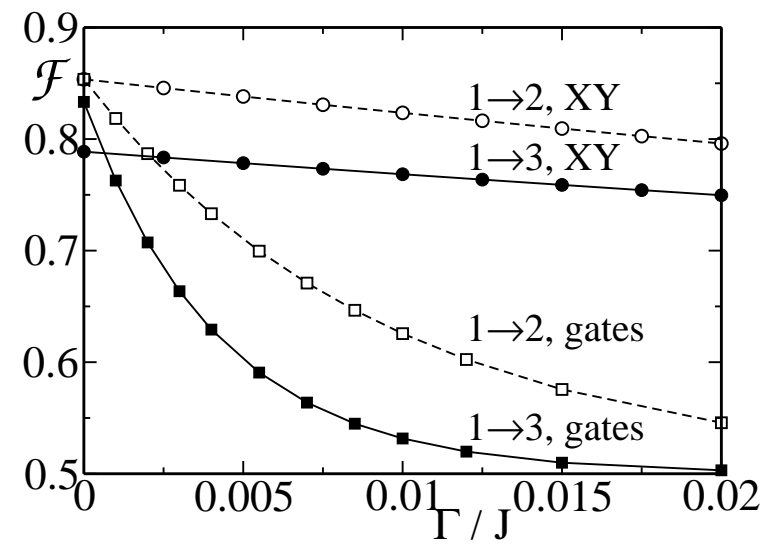

FIG. 3: Comparison of the fidelity $\mathcal{F}_{\lambda}$ obtained by the present method and the quantum circuit discussed in [18, 25] in the presence of an external noise. White and black symbols refer to the $1 \rightarrow 2$ and $1 \rightarrow 3$ case respectively $(\vartheta=\pi / 2$.)

\begin{tabular}{|c|c|c|c|c|c|}
\hline$N$ & $M$ & $\mathcal{F}_{P C C}$ & $\mathcal{F}$ & $J t_{c}$ & $J / B$ \\
\hline 2 & 3 & 0.941 & 0.94 & 81.04 & 99.8 \\
\hline 2 & 4 & 0.933 & 0.90 & 346.75 & 49.0 \\
\hline 2 & 5 & 0.912 & 0.87 & 73.66 & 95.6 \\
\hline 2 & 6 & 0.908 & 0.83 & 277.59 & 70.0 \\
\hline 2 & 7 & 0.898 & 0.81 & 69.04 & 17.6 \\
\hline 3 & 4 & 0.973 & 0.97 & 581.07 & 17.2 \\
\hline 4 & 5 & 0.987 & 0.97 & 584.65 & 57.0 \\
\hline
\end{tabular}

TABLE I: The maximum fidelity $\mathcal{F}$ for $N \rightarrow M$ for the network of figure 1. $\mathcal{F}_{P C C}$ is the optimal fidelity for the PCC 24]. Column 5 (6) reports the corresponding evolution time $t_{c}$ (interaction strength $J$ ). The results refer to the XY model $(\lambda=0)$. The value $\mathcal{F}$ is found by numerical maximization in the intervals $J / B \in[0 ; 100]$ for $N+M<9$ and $J / B \in[0 ; 60]$ for $N+M=9$ and $J t \in\left[0 ; 3 \cdot 10^{3}\right]$. 